\title{
Die Welt als Bedrohung. Der Ausweg heißt nachhaltige Entwicklung
}

Der Einladungstext zur Tagung „Die Welt als Bedrohung. Der Ausweg heißt nachhaltige Entwicklung "1 stellte mehrere Anforderungen an entwicklungspolitische Kommunikation. Sie solle erreichen, dass sich die deutsche Bevölkerung mit internationaler Politik, Globalisierung und Themen der Nachhaltigkeit auseinandersetzte. Als inhaltlicher und politischer Rahmen wird dafür die Agenda 2030 für nachhaltige Entwicklung gesetzt. Nicht nur die Auseinandersetzung, auch Engagement für die darin enthaltenen Nachhaltigkeitsziele solle angestoßen werden. Die bisherige Kommunikation habe keine ausreichende Wirkung entfaltet. Ein Grund bestehe darin, dass in der Bevölkerung Ängste vor der „Welt außerhalb Deutschlands“ und vor eigener Armut bestünden. Entwicklungspolitische Kommunikation könne mehr Erfolge erzielen, wenn sie die Vermittlung globaler Themen und die Anregung zum Engagement an regionalen Erfordernissen ausrichte. Die Teilnehmenden besprachen diese Punkte anhand von Inputreferaten und formulierten Ideen, wie entwicklungspolitische Kommunikation in der Zukunft gestaltet werden könnte.

Um ein Verständnis für mangelndes Interesse und Engagement aus weiten Teilen der Bevölkerung zu entwickeln, wurde die Perspektive der aufgeklärten Apokalyptik herangezogen. Sie erklärt, dass Angst und Hoffnung in ihrem Zusammenspiel ihre jeweils lähmende Wirkung verlören. Während Angst ohne Hoffnung eine Schockstarre hervorrufe, halte eine falsche Hoffnung, es werde sich schon alles richten, ebenso vom Handeln ab. Entwicklungspolitische Kommunikation müsse Bedrohungsszenarien aufmachen, um Handeln anzuregen. Dies müsse davon begleitet werden, dass Einflussmöglichkeiten und positive Zukunftsszenarien aufgezeigt würden: Angst entfachen, die zum Handeln drängt und Hoffnung säen, die Handeln ermöglicht, ohne unmittelbare Ergebnisse sehen zu müssen.

Entwicklungspolitische Kommunikation brauche klare Botschaften. Sie müsse komplexe Befunde verständlich darstellen und auch auf emotionaler Ebene funktionieren. Emotionalisierende Botschaften greifen jedoch häufig auf diskriminierende Bilder und Sprache zurück. Eine Möglichkeit, dem entgegenzuwirken und dennoch an der Erlebniswelt der Angesprochenen anzuknüpfen, sei der Alltagsbezug. Die Situation in Deutschland bietet sowohl für Katastrophenszenarien (Situation in Geflüchtetenunterkünften, rassistische Morde, Waldbrände, Artensterben) als auch für Beispiele entwicklungspolitischen Engagements (kommunale Projekte, Fridays for Future, Bauernproteste) ausreichend Material. Es gelte, auf den Alltagsbezug globaler Themen zu setzen, eigene Handlungsmacht aufzuzeigen und so zu Engagement zu ermutigen.
Wenn es zu bürgerschaftlichem Engagement kommt, darf dieses nicht ins Leere laufen. Politische Prozesse und Strukturen müssen transparent gemacht und erklärt werden. Die Zusammenarbeit an konkreten Projekten bietet einen guten Ausgangspunkt, um übergreifende Themen zu besprechen und gegenseitiges Verständnis zwischen verschiedenen Akteuren herzustellen.

Auf der Suche nach Partnern muss sich entwicklungspolitische Kommunikation ihrer eigenen Voraussetzungen und Interessen bewusst sein. So teilt sie zum Beispiel Inhalte und Methoden mit der politischen Bildung. Wie es das Stichwort Kommunikation jedoch schon sagt, bleibt sie nicht beim Bildungsanspruch. Wo politische Bildung zum Handeln befähigen will, geht entwicklungspolitische Kommunikation weiter und sieht als eines ihrer Ziele, Handeln zur gesellschaftlichen Veränderung anzustoßen.

Entwicklungspolitische Kommunikation hat verschiedene Aufgaben zu erfüllen. Bildungsarbeit, die grundlegendes Verständnis für entwicklungspolitische Themen erzeugt, steht neben politischer Lobbyarbeit, die entwicklungspolitischen Zielen zum Erfolg verhelfen soll. Vermittelnde und koordinierende Kommunikation zwischen entwicklungspolitischen Akteurinnen auf verschiedenen Ebenen und mit Partnern aus anderen Bereichen ist gefragt. Weiter gilt es, die Arbeit und Anliegen entwicklungspolitischer Akteure in der Breite der Bevölkerung bekannt zu machen.

Die Dokumentation zur Tagung erschien im März 2020 und kann beim World University Service (infostelle@ wusgermany.de) angefordert werden.

\section{Anmerkungen}

1 Tagung zu entwicklungspolitischer Kommunikation in Berlin vom 12. bis einschließlich 13. Dezember 2019 auf Einladung des Ministeriums der Justiz und für Europa und Verbraucherschutz des Landes Brandenburg (ehemals), des Vereins zur Förderung von Wissenschaft und Praxis der Mediation, des World University Service und der Evangelischen Akademie zu Berlin

Anne-Sophie Tramer, World University Service - Deutsches Komitee e. V., Informationsstelle Bildungsauftrag Nord-Süd doi.org/10.31244/zep.2020.04.09 